\title{
Research on the Application of Carbon Foam Absorbing Materials in Wireless Testing of Spacecraft Thermal Vacuum Test
}

\author{
DU Chun-lina, YIN Xiao-fang, QIN Jia-yong, LIU Xiao-ning, SUN Yu-wei, FENG-Yao \\ Beijing Institute of Space Environment Engineering, Beijing, 100094
}

\begin{abstract}
With the rise of mobile communication satellites and low-orbit Internet satellite constellations, wireless testing is required in spacecraft thermal vacuum test, and a comprehensive thermal testing environment for vacuum low-temperature absorption is needed. This paper clarifies the key parameters that need to be paid attention to in the application of vacuum absorbing materials under vacuum and low temperature environment, gives reference indexes, and puts forward the technical process and test results for the development of absorbing shroud.
\end{abstract}

Keywords: carbon foam; thermal test; wireless test; absorbing shroud

\section{Preface}

The traditional vacuum thermal test of domestic spacecraft uses wired testing, that is, the satellite payload is connected to high-frequency or low-frequency cables, which is led out of the container through a sealed vacuum flange, and connected to ground testing equipment to complete the test. With the development of satellite antenna technology and the rise of low-orbit Internet satellite constellations in recent years, the development process proposes wireless testing during the vacuum thermal test of satellites to achieve test coverage and effectiveness. Because of this demand, it is necessary to establish a absorbing environment in a vacuum low temperature environment, and the absorbing environment must also cover the frequency band required for satellite testing.

This paper analyzes the key parameters that need to be paid attention to when the absorbing material is used in the vacuum low temperature environment, proposes the corresponding reference index requirements, and introduces the use of the absorbing material based on the work that has been carried out.

\section{Introduction to absorbing materials}

The basic principle of absorbing materials is to convert electromagnetic waves into other forms of energy such as thermal energy, electrical energy or mechanical energy and consume them. It finds and uses the radar stealth technology. As the core part of radar stealth technology, the absorbing material has become a hotspot in the field of military materials because of its relatively simple technology and relatively low design difficulty ${ }^{[1][2][3]}$. In addition to the military stealth field, absorbing materials are also key materials in the fields of defense military and civil technology, such as microwave darkroom, electromagnetic information leakage protection, electromagnetic radiation protection, building absorbing materials, etc. ${ }^{[4]}$

The absorbing material is generally composed of a matrix material (or a binder) and an absorbing medium (absorbent). The binder is the base material to ensure the mechanical properties of the material; the absorbent is the loss medium, which is the key component that determines the absorption performance of the absorbing material. The performance, quantity and matching selection of the absorbing material are the main links in the design of the absorbing material ${ }^{[5]}$. There are many classification methods of absorbing materials. According to their loss mechanism, they can be divided into dielectric loss type absorbing materials and magnetic loss type absorbing materials. According to their functional configuration, they can be divided into coating type and structural type [6].

\section{Application technology process}

\subsection{Determination of performance parameters}

The absorbing materials used in radar stealth technology must meet the requirements of "thin, light, wide, strong" and corrosion resistance ${ }^{[1]}$. The ideal absorbing material should have the characteristics of absorption frequency bandwidth, light weight, thin thickness, good physical and mechanical properties, easy to use, etc. ${ }^{[7][8]}$ However, in the vacuum low temperature environment, whether the absorbing material for wireless testing of spacecraft needs to be thin, wide, light and strong, the analysis is as follows:

\footnotetext{
a Corresponding author: to_cl2004@126.com
} 
(1) The structure of the current wave absorbing material is a pyramid structure, which belongs to the structure type rather than the coating type. The wave absorbing material as the test tool does not contact the test product, so it can have a certain height;

(2) satellites generally involve antennas of different frequency bands and bandwidths, and the frequency bands of different satellites are not the same, so the absorbing material only needs to meet the reflectivity requirements of the corresponding frequency band of the satellite, so the "wide" frequency band may not be used as a key parameter;

(3) During the test, the absorbing material is static and can be temperature controlled, so the structural strength is not high;

(4) The absorbing material is generally installed through a metal frame. Light weight can reduce the degeneration of the metal frame under the alternating temperature environment, and thus maintain the stability of the absorbing device's absorbing performance. Therefore, "light" can be used as a key parameter.

Based on the above analysis, it is known that reflectance and density are the key parameters that need to be considered when the absorbing material is used in a vacuum and low temperature environment. In addition, environmental adaptability should also be considered: such as condensable volatiles, water vapor reabsorption rate, surface emissivity, Vacuum quality loss and other parameters. According to experience, the key parameter reference indexes of recommended absorbing materials under vacuum and low temperature environment are shown in Table 1.

Table 1 Reference parameters of key parameters of absorbing materials

\begin{tabular}{|c|c|}
\hline Key parameters & $\begin{array}{c}\text { Reference } \\
\text { indicators }\end{array}$ \\
\hline Reflectivity & less than $-25 \mathrm{~dB}$ \\
\hline Density & less than $3 \mathrm{~g} / \mathrm{cm}^{3}$ \\
\hline $\begin{array}{c}\text { Condensable Volatile Matter } \\
\text { (CVCM) }\end{array}$ & less than $0.1 \%$ \\
\hline Moisture Vapor Absorption Rate & Less than $1 \%$ \\
\hline Surface emissivity & greater than 0.85 \\
\hline Vacuum quality loss (TML) & less than $2 \%$ \\
\hline
\end{tabular}

\subsection{Performance testing and selection of absorbing materials}

When selecting the absorbing material, the indicators of performance parameters need to be tested by authoritative organizations. Based on previous literature research and performance test results, two materials, carbon foam and silicon carbide, were initially selected; each material has two structural forms, open-cell foam and closed-cell foam. At the same time, the mesh structure of the foam material is also different. Therefore, the selection of materials needs to be based on the test environment. In this project, considering the low-temperature vacuum environment, the materials cannot be closed, so the open-cell foam material is selected, and the cost is also considered. Therefore, this project chooses Open-cell carbon foam materials are used as research objects. Carbon foam materials (hereinafter referred to as "carbon foam") are structural and dielectric loss-type absorbing materials.

The key parameters of carbon foam materials are as follows: the corresponding frequency reflectance is $-28 \mathrm{~dB}$, the density is less than $0.5 \mathrm{~g} / \mathrm{cm}^{3}$, the condensable volatiles are less than $0.01 \%$, the water vapor reabsorption is $0.85 \%$, the surface emissivity is 0.896 , and the vacuum quality loss is $1.39 \%$. In addition, the thermal conductivity of the material is also a parameter that needs attention. The thermal conductivity of carbon foam materials is $0.45 \mathrm{~W} / \mathrm{K} \cdot \mathrm{m}$.

\subsection{Development and test of absorbing shroud}

After the materials are selected, the structural form of the absorbing material is determined, and the assembly process is cured. The principle prototype of the absorbing device is developed for testing the absorbing performance in a vacuum low temperature environment. If the verification test fails, you need to improve the principle prototype and test again. After the test meets the requirements, develop the absorbing device required for the formal test and carry out the formal test.

\section{Carbon foam structure shaping}

An open-cell carbon foam absorber is shown in Figure 1. Carbon foam is prepared by preform molding and high-temperature pyrolysis process. The pyrolysis process is used to control the electrical conductivity of the carbon foam, thereby regulating the electromagnetic parameters. Carbon foam has excellent broadband frequency response characteristics, and its $1-18 \mathrm{GHz}$ broadband electromagnetic parameters are shown in Figure 2

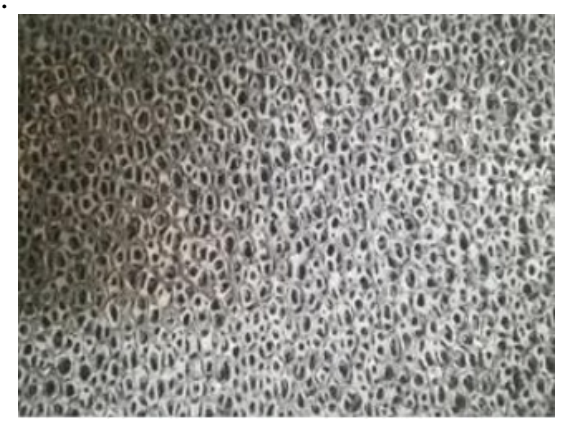

Figure 1. Open-cell carbon foam absorbing material

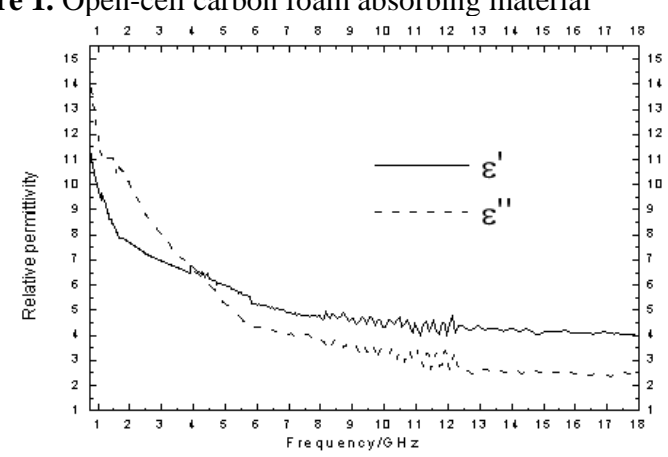

Figure 2. Broadband electromagnetic parameters of open-cell carbon foam 
According to Maxwell's equation, two basic parameters, that is, the dielectric parameter $\varepsilon$ and the permeability $\mu$, can be used to characterize the electromagnetic properties of materials. These two parameters are complex numbers. Carbon foam is a dielectric loss-type absorbing material, so it is important to evaluate the dielectric parameter $\varepsilon$. Under alternating electromagnetic field conditions:

$$
\varepsilon=\varepsilon^{\prime}-i \varepsilon^{\prime \prime}
$$

In the formula: $\mathrm{i}$ is an imaginary unit, and the relative dielectric constant $\mathrm{gr}$ is generally used to characterize the properties of materials in engineering.

$$
\varepsilon_{r}=\frac{\varepsilon}{\varepsilon_{0}}=\varepsilon_{r}-i \varepsilon_{r}
$$

Where $\varepsilon 0$ is the dielectric constant parameter value of the material in free space.

The real and imaginary parts of the dielectric constant have specific physical meanings: the real part can characterize the degree of polarization of a material under the action of an electric field, and the imaginary part is a measure of the energy dissipated when a material is polarized in an electric field; The electromagnetic parameters of wave materials are often closely related to the composition, structure size, and micro-morphology of the wave absorbing material. Absorbing materials have contradictory relations in terms of increasing electromagnetic losses and meeting impedance matching conditions $^{[8]}$. Based on the above electromagnetic parameters, the electromagnetic simulation design of carbon foam was carried out, and the specific dimensions of the integrated combined carbon foam pyramid were determined, as shown in Figure 3: flat carbon foam A with a thickness of $14 \mathrm{~mm}$; and the size of the bottom side of the sharp carbon foam B is $50 \mathrm{~mm} \times 50 \mathrm{~mm}$, the size of top side is $5 \mathrm{~mm} \times 5 \mathrm{~mm}$ and $70 \mathrm{~mm}$ high.

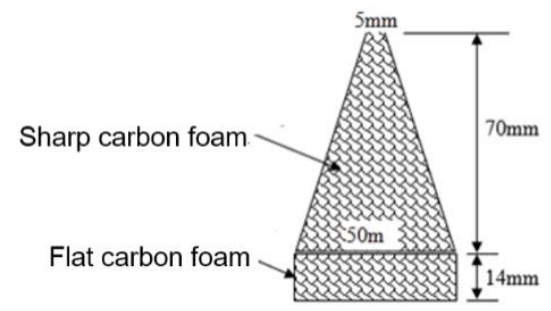

Figure 3. Simulation model of carbon foam composite structure and specific dimensions

The actual measurement results of the carbon foam absorbing performance provided by the third-party identification department are shown in Figure 4. Both the simulation and the actual measurement results show that the carbon foam absorbing performance is better than $-28 \mathrm{~dB}$ in the test required frequency band.

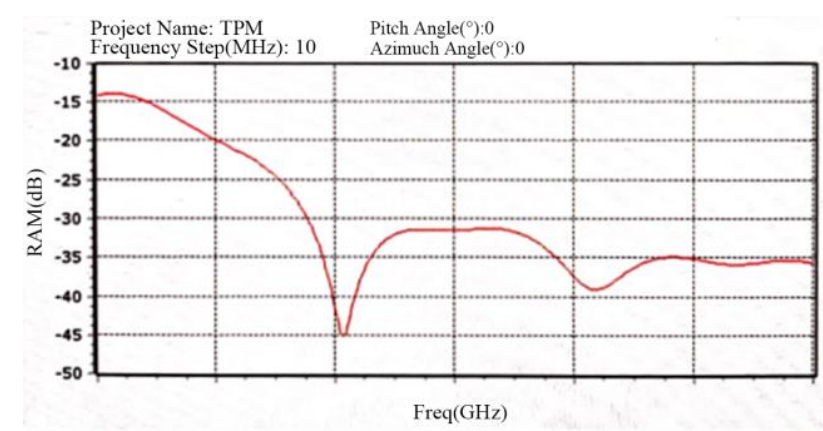

Figure 4. Third-party test results of the integrated carbon foam pyramidal wave-absorbing performance

\section{Test}

The shaped carbon foam pyramid was used to develop a heat-absorbing shroud, as shown in Figure 5. During the test, the absorbing shroud is placed in a container, and the two feed antennas are fixed inside the absorbing device with a non-metal plate. The high-frequency cable leads out of the cabin and connects to the test equipment, as shown in Figure 6. ${ }^{[9]}$

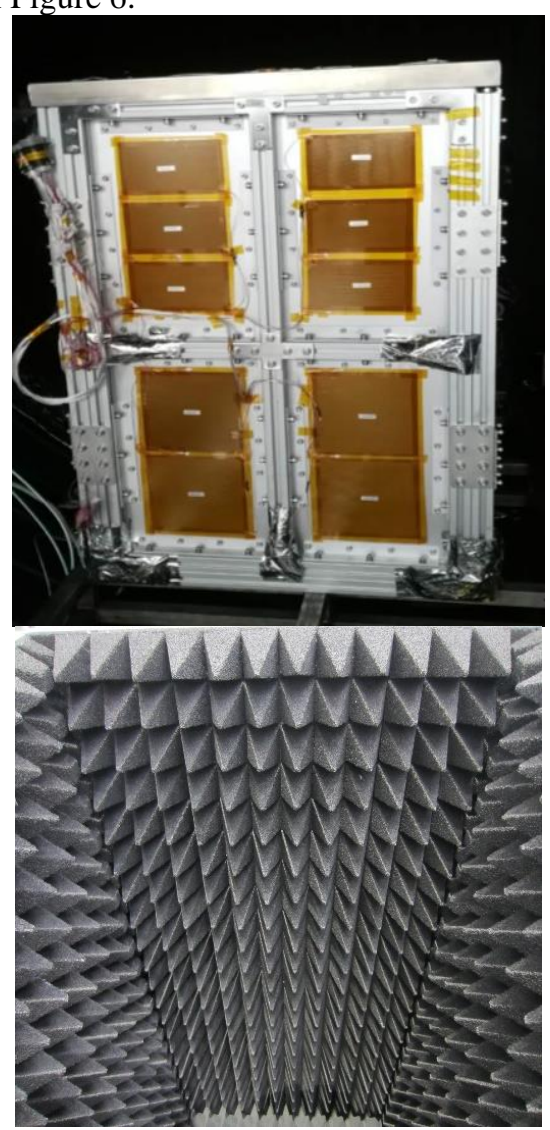

Figure 5. Test state of absorbing shroud 

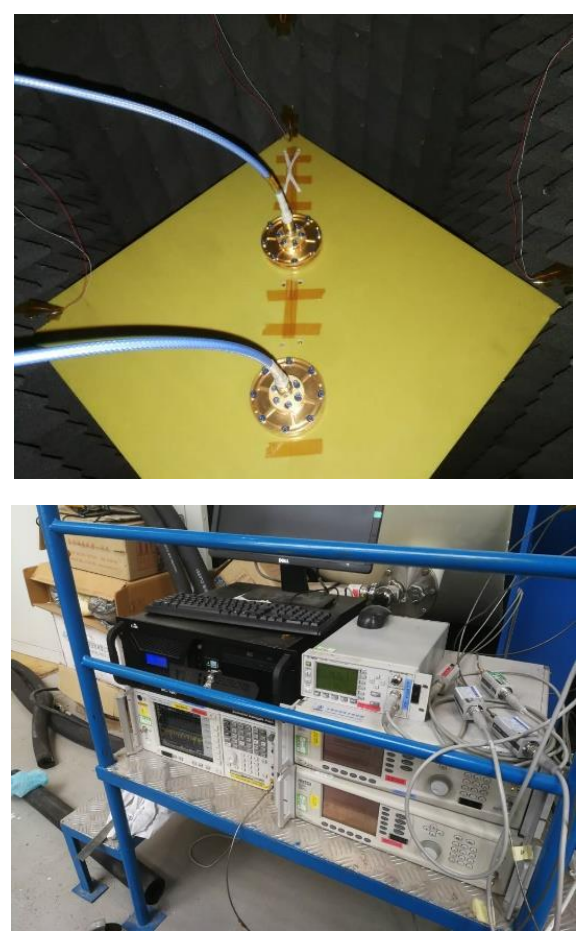

Figure 6. Test status and test equipment of the feed

The application requirements of this time absorbing material are: the reflectance within the specified frequency range of the test is $\leq-25 \mathrm{~dB}$, and the passive intermodulation (PIM) level indicator is less than $-150 \mathrm{dBm}$ in the required power mode.

The test results in a low-temperature vacuum environment show that the carbon foam pyramid and the installation process can meet the requirements for use in a vacuum environment. The temperature of the internal pyramid surface is controllable, and the results of the temperature control of the absorbing shroud are shown in Figures 7 and 8 . It can be seen that the internal and external temperature trends of the wave absorbing shroud are basically the same. At the high temperature equilibrium stage, the internal and external temperature difference is about $20^{\circ} \mathrm{C}$ (high inside).

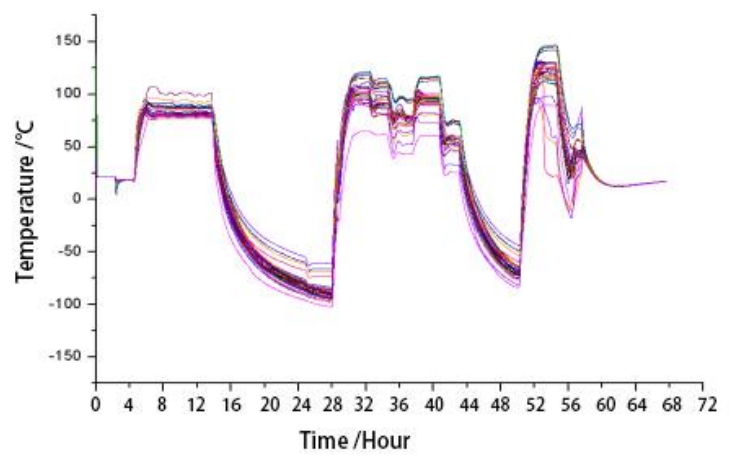

Figure 7. Temperature control results of absorbing shroud

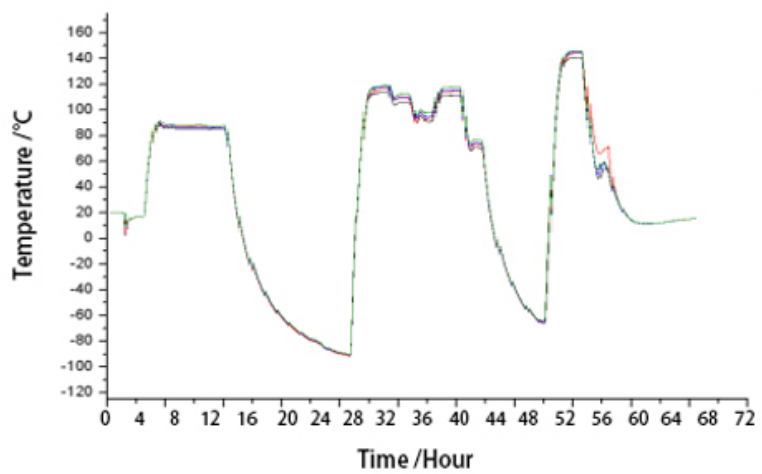

Figure 8. Temperature data of the external temperature measurement point of the wave absorbing shroud

The absorbing shroud was tested in a vacuum and low temperature environment for 5 days. During this period, the absorbing index PIM level test device has been turned on for testing. The test results are shown in Figure 9. It can be found in the range of $-60{ }^{\circ} \mathrm{C} \sim+90^{\circ} \mathrm{C}$ The PIM level is better than $-150 \mathrm{dBM}$. When the temperature of the absorbing box reaches $100{ }^{\circ} \mathrm{C}$ (the internal cavity temperature is about $115^{\circ} \mathrm{C} \sim 119{ }^{\circ} \mathrm{C}$ ), a sudden peak of the absorbing index appears, reaching a maximum of $-138 \mathrm{dBM}$ for 4 seconds. It did not reappear after that, and it did not affect the test conclusion.

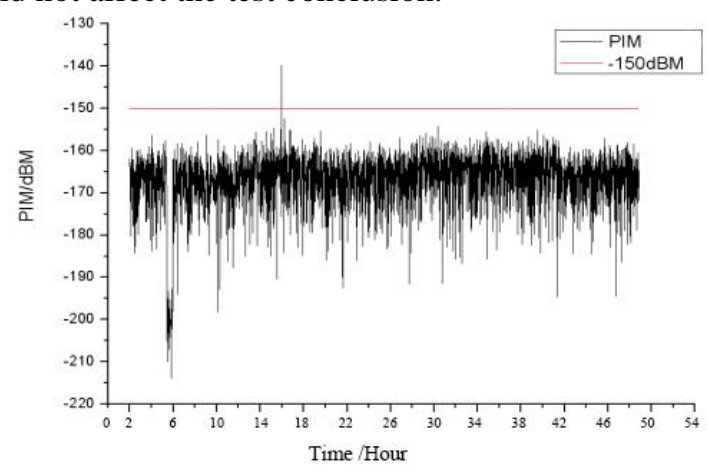

Figure 9. PIM level of the absorption shroud during the test

\section{Conclusion}

Absorbing materials are mostly porous materials and may contain organic components. These are factors that are not conducive to use in a vacuum and low temperature environment. Based on many years of experience, this paper gives the key parameters and reference indicators that need to be considered when using the absorbing material in a vacuum and low temperature environment. The development process and actual test results can provide references for subsequent use. It must be noted that wireless testing of spacecraft in a vacuum and low temperature environment requires different concerns. For example, if good thermal control uniformity is required, the thermal conductivity of the absorbing material should be paid attention to; Must strictly control the assembly process; the use of pyramidal absorber structure also needs to consider broken cones and excess issues. 


\section{References}

1. Luo Fa, Zhou Wancheng, Jiao Yan, et al. Research status of high temperature absorbing materials [J], Aerospace Materials Technology, 2002, (1), 8-11;

2. F. Moglie, D. Micheli, S. Laurenzi, M. Marchetti and V. P. Mariani, "Electromagnetic Shielding Performance of Carbon Foams," Carbon, Vol. 50, No. 5, 2012, pp. 1972-1980. doi: 10.1016/j.carbon.2011.12.053;

3. D. Micheli, C. Apollo, R. Pastore and M. Marchetti, "XBand Microwave Characterization of Carbon-Based Nanocomposite Material, Absorption Capability Comparison and RAS Design Simulation," Composites Science and Technology, Elsevier, Vol. 70, No. 2, 2010, pp. 400-409;

4. Hu Xiaosai, Shen Yong, Wang Liming, et al.Study on the structure, properties and application of microwave absorbing materials[J], Applied Chemical Industry, 2015, 44 (9): 1741-1746;
5. Wang Zupeng, Yu Mingxun, Pan Bingbing, et al. Development in study on fiber absorbing materials [J], New Chemical Materials, 2010,38 (4): 13-15;

6. Cui Zhiping, Li Xiaobo, Research Progress of Absorbing Materials [J], China Chemical Industry and Trade, 2011, 44 (1): 1-5;

7. Pang Jianfeng, Ma Xijun, Xie Xingyong, Research progress of electromagnetic absorbing materials $[\mathrm{J}]$, Electronic Components and Materials, 2015, 34 (2): 7-12;

8. Hou Yongshen, Wang Jianjiang, Xu Baocai, et al. Application Status and Research Progress on Wave Absorbing Materials Based on Computer Aided Design Technology [J], Materials Review A: Overview 2014, 28 (1): 127-131;

9. DU Chun-lin, QIN Jia-yong, YIN Xiao-fang, et al. Research on Microwave load Wireless PIM test of spacecraft in thermal vacuum environment [J]. Spacecraft Environment Engineering, 2017,34 (4): 370-375 